\title{
Totally Laparoscopic Distal Gastrectomy in Post Liver Transplant Patient
}

\author{
Eun Young Park, M.D. ${ }^{1}$, Dong Jin Park, M.D., M.S. ${ }^{1,2}$, Hyung Woo Park, M.D., M.S. ${ }^{1,2}$, Chang Woo Nam, M.D., Ph.D. ${ }^{1,2}$,
} Yang Won Nah, M.D., Ph.D. ${ }^{1,2}$, Gyu Yeol Kim, M.D., Ph.D. ${ }^{1,2}$

Department of Surgery, 'Ulsan University Hospital, ${ }^{2}$ University of Ulsan College of Medicine, Ulsan, Korea

\begin{abstract}
The risk of malignancy after transplantation is higher than that of general population. Laparoscopic surgery has become a standard treatment of gastric cancer. However, there are no case reports evaluating totally laparoscopic gastrectomy in patients with previous liver transplantation. Herein we report our experience with a liver transplant recipient who underwent totally laparoscopic distal gastrectomy (TLDG) for gastric cancer. A 63 year-old man underwent orthotopic liver transplantation (OLT) for cryptogenic liver cirrhosis. 8 years later, gastric cancer was diagnosed during the follow-up. Endoscopic submucosal dissection was performed and additional surgical resection was needed. TLDG and D1+ lymph node dissection was performed, and the patient was discharged on the 8th post-operative day without any complications. To the best of our knowledge, this is the first case of de novo gastric cancer treated with TLDG after OLT. This suggests that TLDG is a feasible for patients after OLT.
\end{abstract}

Keywords: Gastrectomy, Laparoscopy, Gastric cancer, Liver transplantation
Received June 19, 2018

Revised July 12, 2018

Accepted July 13, 2018

Corresponding author

Dong Jin Park

Department of Surgery, Ulsan

University Hospital, 877

Bangeojinsunhwando-ro, Dong-gu,

Ulsan 44033, Korea

Tel: $+82-52-250-7000$

Fax: $+82-52-250-8071$

E-mail: parkdongjin7907@gmail.com ORCID:

http://orcid.org/0000-0001-7820-9179

Copyright @ 2019 The Journal of Minimally Invasive Surgery. All rights reserved.

This is an Open Access article distributed under the terms of the Creative Commons Attribution Non-Commercial License (http:/ creativecommons.org/licenses/by-nc/4.0/) which permits unrestricted non-commercial use, distribution, and reproduction in any medium, provided the original work is properly cited.

\section{INTRODUCTION}

Long-term survival after liver transplantation has significantly improved with advances in immunosuppressive therapy and surgical techniques. However, prolonged immunosuppression has been reported to increase the risk of de novo malignancy. ${ }^{1}$ These malignancies have become an important cause of death after transplantation. ${ }^{2}$ The reported incidence of de novo malignancy following liver transplantation ranges from $2 \%$ to $14.4 \% .^{3-5}$ Unlike other countries, it is recognized that the most common de novo malignancy associated with liver transplantation is gastric cancer in Korea. ${ }^{3}$

Laparoscopic surgery has become a standard approach in the treatment of early gastric cancer. ${ }^{6}$ Likewise, the use of laparoscopy is markedly increasing in the field of liver trans- plantation. In 2011, the first report was published regarding laparoscopy assisted distal gastrectomy to be a feasible method for gastric cancer treatment in patient with previous liver transplantation. ${ }^{8}$ However, there have been no more reports since then. To our knowledge, there is no case report evaluating the feasibility of totally laparoscopic gastric cancer surgeries in liver transplant recipients. Herein we report our experience with a liver transplant recipient who underwent totally laparoscopic distal gastrectomy (TLDG) for gastric cancer.

\section{CASE REPORT}

A 63 year-old man underwent orthotopic liver transplantation (OLT) for cryptogenic liver cirrhosis at our hospital in August 2009. He recovered without any complications. After 
the transplantation, Tacrolimus (for one month), prednisolone (for three months) and mycophenolate mofetil (for twentyseven months) were administered for immunosuppression. Currently, the patient is receiving cyclosporin A alone. In November 2017, esophagogastroduodenoscopy (EGD) was performed as a regular screening and gastric cancer was diagnosed incidentally. The lesion was located in the proximal antrum and measured $1.8 \mathrm{~cm}$ (Fig. 1); it was histologically welldifferentiated adenocarcinoma. The depth of invasion in the gastric wall was estimated mucosal layer and no other distant metastasis or de novo malignancy was recognized by further examination. Endoscopic submucosal dissection (ESD) was performed, after which the biopsy result showed incomplete resection margin, undifferentiated histologic type and lymphovascular invasion; it was indication for surgery.

Totally laparoscopic distal gastrectomy (TLDG) and D1+

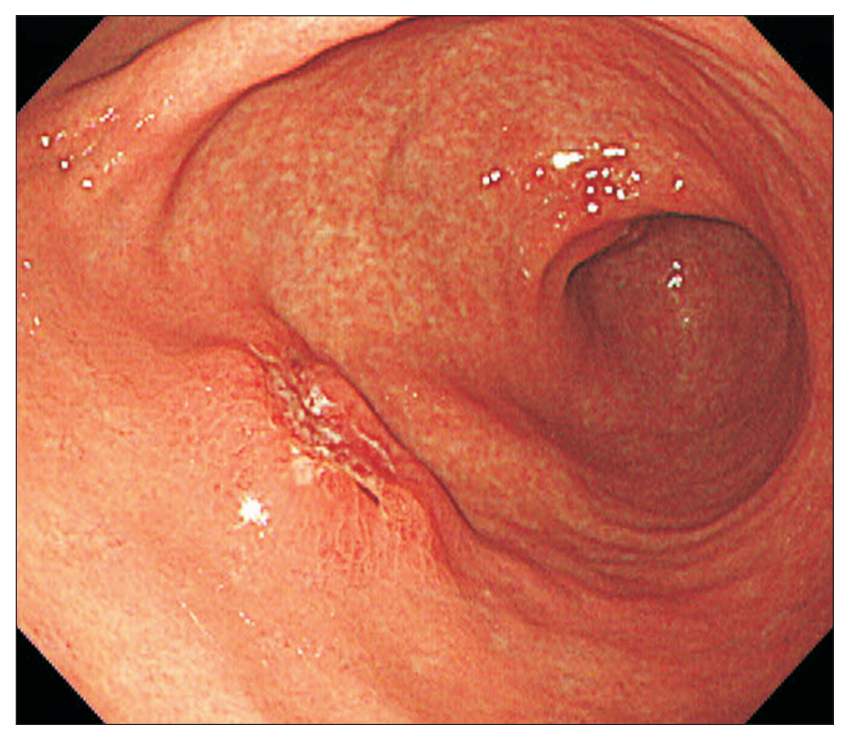

lymph node dissection was performed in December 2017 (Fig. $2 \mathrm{~A}, \mathrm{~B}$. Understanding the previous OLT is very important to avoid possible damaging the transplanted liver during gastrectomy. Therefore, consults from the liver transplant surgeons were seeked before operation. The patient was laid supine under general anesthesia. Mercedes-Benz incision had been used for the previous OLT. For the laparoscopic surgery, a 12 $\mathrm{mm}$ trocar was carefully inserted through an umbilical incision using the Hasson open technique. Severe adhesions were present at upper abdomen. However, there was no evidence of metastasis. Additional right $12 \mathrm{~mm}$ and left $5 \mathrm{~mm}$ trocars were inserted at both mid-clavicular line $3 \mathrm{~cm}$ above the umbilicus. After carefully dissecting the adhesions so that the extra trocars could be inserted safely, two $5 \mathrm{~mm}$ trocars were inserted at both mid-axillary lines below the costal margin. The integrated bipolar and ultrasonic energy device (Thunderbeat ${ }^{\mathrm{TM}}$, Olympus, Japan) was used for dissection of adhesions and lymph nodes. Dissection of lymph nodes around the common hepatic artery was extremely challenging due to anatomical changes and severe adhesions after the OLT (Fig. 2C). However, other surgical procedures did not differ from those of usual TLDGs. The umbilical trocar site was extended to $4 \mathrm{~cm}$ vertically after distal gastrectomy; the specimen (Fig. 3) was extracted through this incision and intracorporeal gastrojejunostomy was performed. Operation time was 305 minutes, and the estimated blood loss was $200 \mathrm{ml}$.

After the surgery, routine post-operative care was performed and the adequate immunosuppressive medications were administrated. The histopathological examination of the resected stomach revealed no residual malignancy. Total number of retrieved lymph nodes was 22, and one lymph node metastasis was present in lymph node station 7 (pT1bN1M0). The patient recovered well and was discharged on postoperative day 8 without any complications (Fig. 4).

Fig. 1. Endoscopic finding showed early gastric cancer, type Ilc + Ila lesion on anterior wall of the gastric antrum.
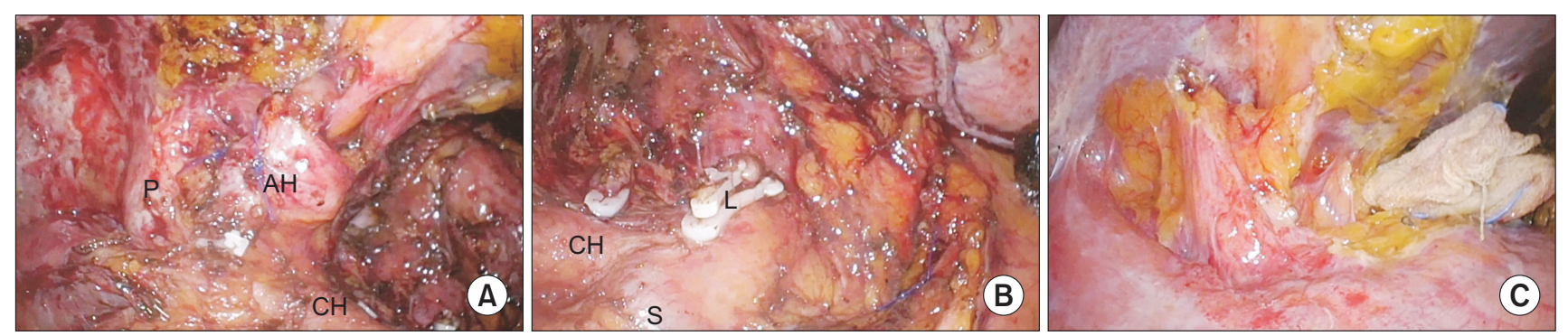

Fig. 2. (A) Intraoperative view after dissection of the D1 + lymph node around the hepatoduodenal ligament. (B) Intraoperative view after dissection of the D1 + Iymph node around the celiac axis. (C) Intraoperative view of hepatoduodenal ligament that can not distinguish anatomical structures due to very severe adhesions. $\mathrm{P}=$ portal vein; $\mathrm{AH}=$ anastomosed hepatic artery; $\mathrm{CH}=$ common hepatic artery; $\mathrm{S}=$ splenic artery; $\mathrm{L}=$ divided left gastric artery. 


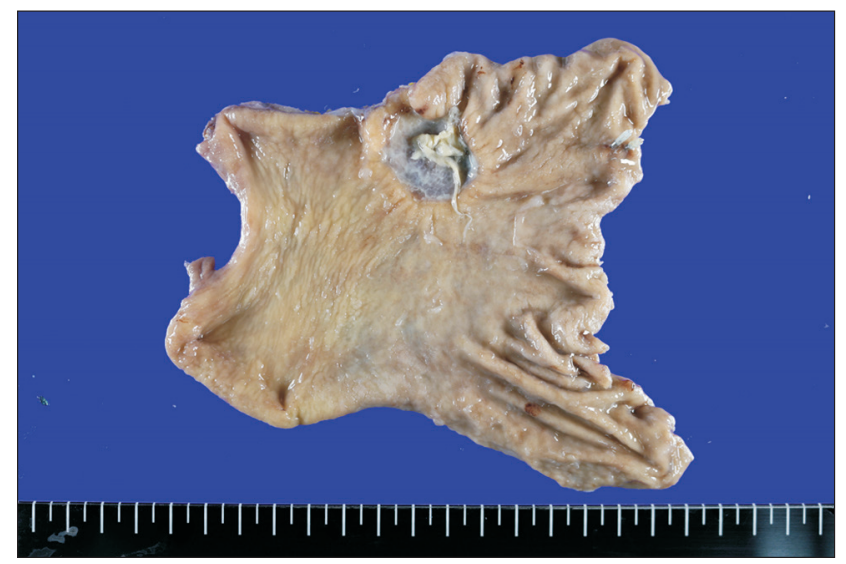

Fig. 3. There was a well demarcated, ulcerative lesion $(3.0 \times 3.0 \mathrm{~cm})$ in the proximal antrum along the anterior wall.

\section{DISCUSSION}

De novo malignancy after organ transplantation has become an important risk factor affecting the long term survival of the recipients. Moreover, the incidence is expected to increase in patients who underwent liver transplantation, so de novo malignancy is anticipated to be the principal cause of death among recipients in the near future.

Previous study has emphasized the necessity for frequent clinical follow-ups for early cancer detection in transplant patients. ${ }^{9}$ Gong et al. showed that the prognosis of de novo gastric cancer in liver transplantation patients was good because the proportion of early gastric cancer was higher than that of advanced gastric cancer. ${ }^{9}$ However, currently there is no widely accepted screening protocol for malignancies after liver transplantation, especially for gastric cancer. In Korea, the National Health Insurance Service recommends EGD once every 2 years for people age 40 and older. Therefore, considering that liver transplant patients have a higher risk of gastric cancer, they should receive routine screening tests, including EGD, at least once a year.

In liver transplant recipients, additional gastric cancer surgery is more difficult due to several factors such as severe post-operative adhesions, changes in anatomy, and the risk of graft loss with vascular injuries. For this reason, almost all patients had been treated with conventional open surgery or ESD. Laparoscopic gastrectomy has several advantages compared with open gastrectomy: less pain, faster recovery of bowel function, and shorter hospital stay. In addition, the long-term oncologic outcomes were comparable. In another study, laparoscopic gastrectomy showed fewer inflammatory and immunologic reactions compared to open gastrectomy. ${ }^{10}$

In contrast to the increased role of diagnostic and therapeu-

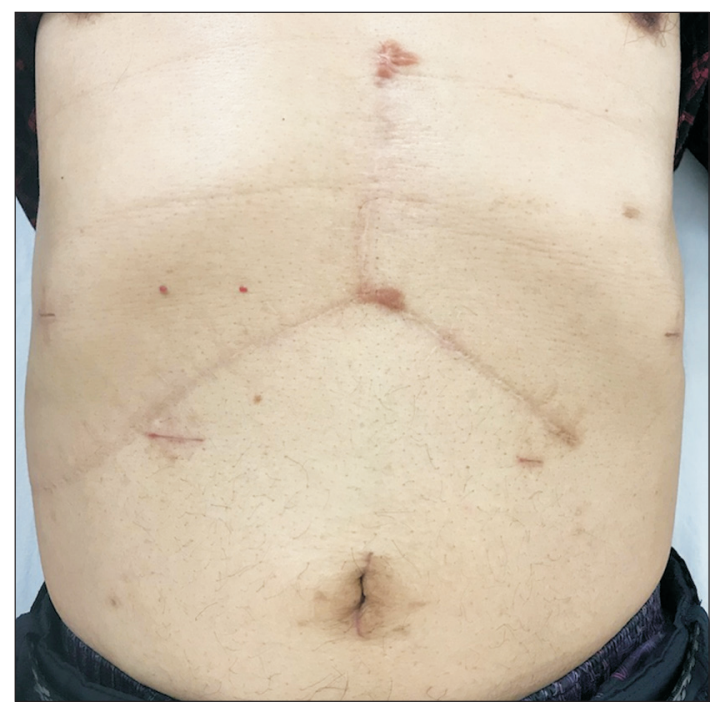

Fig. 4. Post-operative wound after discharge. Previously used MercedesBenz incision and trocar insertion site.

tic laparoscopy in liver transplantation, only one case report was published; laparoscopy assisted distal gastrectomy in liver transplantation patient. No more report has been reported since then.

Laparoscopic gastrectomy after liver transplantation is a difficult procedure. Especially, lymph node dissection around the hepatoduodenal ligament and the hepatic artery must be very carefully performed with accurate anatomical information. With development of instruments and surgical techniques, several barriers in the field of laparoscopy have been overcome. Although a lot of effort and experiences are needed, laparoscopic gastrectomy eventually will be an alternative to open gastrectomy even in patients with previous liver transplantations. To the best of our knowledge, there are no case reports about TLDG in patients with prior liver transplantation. This is the first case of de novo gastric cancer treated with totally laparoscopic techniques. This suggests that TLDG is a feasible method for patients with liver transplantation requiring surgical therapy for gastric cancer.

\section{REFERENCES}

1) Nagata Y, Eguchi S, Takatsuki M, et al. Experience of gastric cancer in a patient who had received a living-donor liver transplantation. Gastric Cancer 2007;10:187-190.

2) Pillai AA. Management of de novo malignancies after liver transplantation. Transplant Rev (Orlando) 2015;29:38-41.

3) Park HW, Hwang S, Ahn CS, et al. De novo malignancies after liver transplantation: incidence comparison with the Korean cancer registry. Transplant Proc 2012;44:802-805. 
4) Egeli T, Unek T, Ozbilgin M, et al. De Novo Malignancies After Liver Transplantation: A Single Institution Experience. Exp Clin Transplant 2017 Dec 17 [Epub]. DOI: 10.6002/ect.2017.0111.

5) Burra P, Rodriguez-Castro KI. Neoplastic disease after liver transplantation: Focus on de novo neoplasms. World J Gastroenterol 2015;21:8753-8768.

6) Kim HH, Han SU, Kim MC, et al. Long-term results of laparoscopic gastrectomy for gastric cancer: a large-scale case-control and case-matched Korean multicenter study. J Clin Oncol 2014; 32:627-633.

7) Lai Q, Pinheiro RS, Levi Sandri GB, et al. Laparoscopy in Liver
Transplantation: The Future has Arrived. HPB Surg 2012;2012: 148387.

8) Lee MS, Kim EY, Lee JH, et al. Laparoscopy-assisted distal gastrectomy for gastric cancer after liver transplantation. J Korean Surg Soc 2011;80 Suppl 1:S1-5.

9) Gong CS, Yoo MW, Kim BS, et al. De Novo Gastric Cancer After Liver Transplantation. Ann Transplant 2016;21:386-391.

10) Jung IK, Kim MC, Kim KH, Kwak JY, Jung GJ, Kim HH. Cellular and peritoneal immune response after radical laparoscopy-assisted and open gastrectomy for gastric cancer. J Surg Oncol 2008;98:5459. 